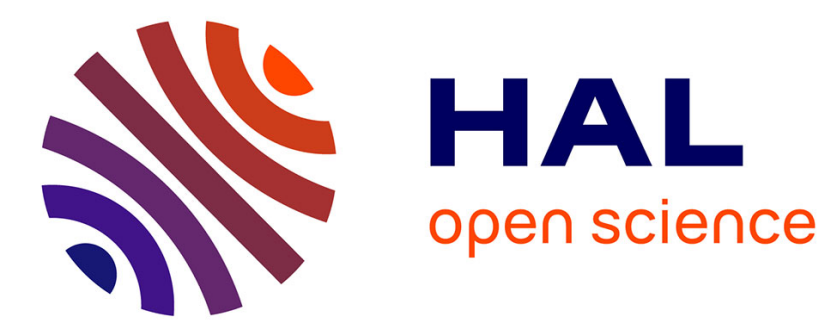

\title{
Evolution of internal stresses in rolled $\mathrm{Zr} 702 \alpha$
}

\author{
David Gloaguen, Marc François, Ronald Guillen, Jean Royer
}

\section{To cite this version:}

David Gloaguen, Marc François, Ronald Guillen, Jean Royer. Evolution of internal stresses in rolled Zr702 $\alpha$. Acta Materialia, 2002, 50 (4), pp.871-880. 10.1016/S1359-6454(01)00380-9 . hal-01006826

\section{HAL Id: hal-01006826 https://hal.science/hal-01006826}

Submitted on 3 Mar 2017

HAL is a multi-disciplinary open access archive for the deposit and dissemination of scientific research documents, whether they are published or not. The documents may come from teaching and research institutions in France or abroad, or from public or private research centers.
L'archive ouverte pluridisciplinaire HAL, est destinée au dépôt et à la diffusion de documents scientifiques de niveau recherche, publiés ou non, émanant des établissements d'enseignement et de recherche français ou étrangers, des laboratoires publics ou privés. 


\title{
Evolution of internal stresses in rolled $\mathrm{Zr} 702 \alpha$
}

\author{
D. Gloaguen a ${ }^{\text {a }}$ M. François ${ }^{\mathrm{b}}$, R. Guillen ${ }^{\mathrm{a}}$, J. Royer ${ }^{\mathrm{c}}$ \\ ${ }^{a}$ Laboratoire d'Applications des Materiaux à la Mecanique_LAMM, I.U.T. Saint-Nazaire, C.R.T.T., B.P 406, 44602 Saint- \\ Nazaire cedex, France \\ ${ }^{\mathrm{b}}$ Laboratoire des Systèmes Mecaniques et d'Ingenierie Simultanee (LASMIS), Universite de Technologie de Troyes, 11 rue Marie \\ Curie, BP2060, 10010 Troyes, France \\ ${ }^{\mathrm{c}}$ Laboratoire Mecanique et Materiaux (LMM), Ecole Centrale de Nantes, 1 rue de la Noë, BP92101, 44321 Nantes cedex 03, \\ France
}

Internal stresses due to anisotropic thermal and plastic properties were investigated in a rolled Zirconium- $\alpha$. The thermal stresses induced by a cooling process were predicted using a self-consistent model and compared with experimental results obtained by X-ray diffraction. The study of the elastoplastic response during uniaxial loading was performed along the rolling and the transverse direction of the sheet, considering the influence of the texture and the thermal stresses on the mechanical behaviour. We used an elastoplastic self-consistent formulation and the predicted results are compared with mechanical tests. The role of twinning and slip on the development of internal stresses is also discussed.

Keywords Zirconium; Elastoplastic self-consistent model; Internal stresses; Thermal anisotropy; X-ray diffraction (XRD)

\section{Introduction}

Zirconium alloys are widely used in the nuclear industry because they exhibit high resistance to corrosion, good thermal conductivity and a low neutron cross section. They have a hexagonal close packed structure at room temperature. These alloys present anisotropic thermal, elastic and plastic properties at mesoscopic (grain) and macroscopic levels. These properties and the crystallographic texture explain the appearance and the development of internal stresses when an elastoplastic deformation is introduced. Two types of internal stresses can be distinguished:

- Thermal internal stresses, which may arise from thermal anisotropy. During the cooling process after a heat treatment, each grain is subjected to a contraction which depends on crystallographic direction. Elastic strain (and this stress) must be present to maintain the compatibility of total strain between adjacent grains -Mechanical internal stresses, induced by the elastoplastic anisotropy and the requirement of compatibility. 
Both kinds of stresses depend on crystallographic texture. Thermal stresses can modify the macroscopic elastoplastic response of the material, particularly for Zirconium alloys. Ortiz et al. [1,2] evaluated these stresses in a textured $\mathrm{Zr}$ sheet using a self consistent model and they compared them with experimental results obtained by X-ray diffraction. They showed that thermal residual stresses produce apparent ("measured") macroscopic stresses that can amount $20 \%$ of the yield stress of the material. Several authors [3-7] performed a systematic analysis of the response of rolled Zircaloy-2 under mechanical loading (tensile and/or compression). They used neutron diffraction techniques in conjonction with a self consistent or a Taylor model. They showed that the plastic anisotropy in both rolling and transverse directions can be attributed to the texture. The residual stresses can explain the stress differential (difference between the yield stresses along the rolling and tranverse directions) in tension and compression and the difference in the elastoplastic transition when Zircaloy-2 is deformed.

The purpose of the present work is to make a more complete study concerning the development and the evolution of residual strain introduced by tensile tests in rolled textured polycrystalline $\mathrm{Zr}-\alpha$ after a cooling process. A self consistent model of elastoplastic deformation was used to simulate the thermal and the tensile response of samples tested along the rolling and the transverse directions of the sheet. The theoretical stresses will be compared with the experimental results obtained by X-ray diffraction and from mechanical tests. The role played by the texture on the development of internal stresses during the cooling process will be analysed. For the calculation of internal stresses during the thermal treatment, we will compare two assumptions: independence of the elastic and thermal constants with the temperature and dependence of these constants with the temperature. The results predicted by the self consistent model will be discussed. In particular, twinning is treated as a directional deformation system and the reorientation by slip and twinning is accounted for in our calculation.

\section{Experiments}

\subsection{Mechanical behaviour}

In the present study, a cold rolled sheet of industrial grade Zirconium $(\mathrm{Zr} 702 \alpha)$ is considered. It presents equiaxed grains with an average grain size of $20 \mu \mathrm{m}$. Its chemical composition is given in Table 1.

To analyse the influence of loading direction and texture on the mechanical response of the material, tensile samples were cut from the rolling and the transverse directions of the sheet. The specimens were submitted to uniaxial tensile tests at room temperature, at a constant strain rate of $3.8 \times 10^{-3}$ $\mathrm{s}^{-1}$ and up to $1 \%$ of total strain. The experimental stress-strain curves are plotted on Fig. 1.

\subsection{Stress analysis}

Even after annealing at room temperature, the material is not stress free at a mesoscopic (grain) scale, due to the anisotropy of thermal behaviour. The thermal residual stresses in the undeformed sample were evaluated using X-ray diffraction technique. After a thermal treatement at $650^{\circ} \mathrm{C}$ during $4.5 \mathrm{~h}$, strain measurements have been carried out on a D500 SIEMENS goniometer with a $\mathrm{Cr} \mathrm{K} \alpha$ radiation. An $\Omega$ goniometric assembly with a scintillation detector were used. Two plane families were studied: $\{10 \overline{1} 4\}$ at $2 \theta=156.7^{\circ}$ and $\{20 \overline{2} 2\}$ at $2 \theta=137.2^{\circ}$. Diffractograms were recorded for fifteen tilt angles $\Psi$ varying between -45 and $45^{\circ}$ and for azimuth angles $\phi=0,45$ and $90^{\circ}$. The direction $\phi=0^{\circ}$ corresponds to the rolling direction. The X-ray beam output collimator had $0.5 \mathrm{~mm}$ diameter and a diffracted-beam slit limits the equatorial divergence to an angle of $0.6^{\circ}$. Peak position is determined by the half value breadth. The stress was obtained from the $\sin ^{2} \Psi$ relation using the macroscopic elasticity constants. Because of the low mesoscopic elastic anisotropy, $\varepsilon_{\phi \Psi}$ vs $\sin ^{2} \Psi$ curves for a given family of diffracting planes $\{h k i l\}$ can be represented by straight lines (Fig. 5). In this case, the XEC can be treated as those corresponding to an isotropic material. The sample shows a typical texture of rolled Zirconium. The basal poles are preferentially 
Table 1

Chemical composition of Zirconium sheet

\begin{tabular}{lllllll}
\hline Element & $\mathrm{O}$ & $\mathrm{C}$ & $\mathrm{Fe}+\mathrm{Cr}$ & $\mathrm{H}$ & $\mathrm{N}$ & $\mathrm{Hf}$ \\
\hline Wt. \% & 0.14 & 0.02 & 0.08 & $<0.0003$ & 0.004 & 0.4 \\
\hline
\end{tabular}

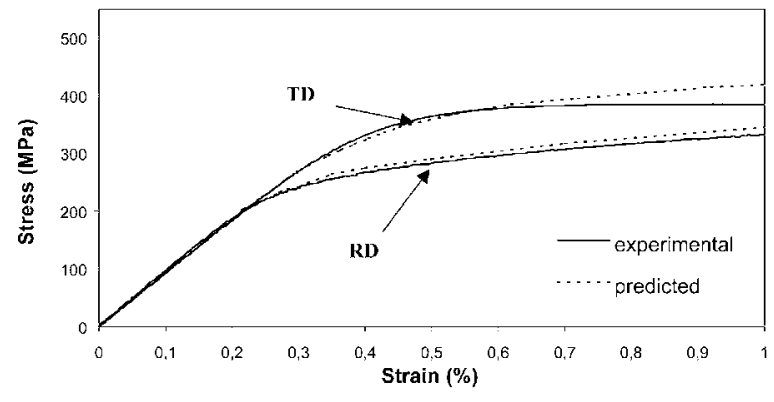

Fig. 1. Experimental and calculated stress-strain curves for tension in the Rolling Direction and Transverse Direction of the sample.

oriented at an angle of $32^{\circ}$ from the normal direction towards the transverse direction, while the prism poles exhibit a weak maximum at the rolling direction.

The experimental data from the mechanical tests and X-ray diffraction are presented and compared with the results from our model in the third and fourth sections.

\section{Self consistent model}

In accordance with self consistent models that were used previously for the prediction of thermal $[6,10]$ and tensile deformations $[8,9]$, we consider that thermal contractions do not introduce plastic deformation in the material during the cooling process [1,2]. In this case, a thermoelastic formulation is sufficient to simulate the evolution of thermal stresses associated with cooling. An elastoplastic self-consistent scheme is then used to predict the mechanical behaviour of zirconium polycrystal under an uniaxial tension.

The self consistent model is based on the following assumptions:

- Each grain is considered as a spherical inclusion embedded in a Homogeneous Effective Medium (HEM) having the average properties of the aggregate.

- The mesoscopic stress $\sigma$ and the mesoscopic strain $\varepsilon$ in the inclusion are homogeneous.

- The overall response of the material is determined from averaging the microscopic terms $(\sigma$, $\varepsilon)$ over a representative set of orientations.

- The macroscopic stress $\Sigma$ and macroscopic strain $\mathbf{E}$ are considered as uniform. The properties of the medium are initially undetermined and must be solved by iterations. Each grain is treated as an inhomogeneity with elastic (c) and thermal ( $\boldsymbol{\alpha})$ constants, slip and twin mechanisms characteristic of a single crystal.

- The texture is known, the polycrystal is represented by a weigthed discrete distribution of orientations (Euler angles $\left.\left(\varphi_{1} \phi \varphi_{2}\right)\right)$. Lattice rotation (reorientation by slip and twinning) and texture changes are incorporated in the model.

We present succinctly the model used for our simulations. More details can be found in references [6,8-10].

\subsection{Simulation of cooling process}

When the polycrystal is subjected to a uniform temperature increment rate $\dot{T}$ and a macroscopic stress rate $\dot{\Sigma}$, the total strain rate in each grain is given by:

$\dot{\boldsymbol{\varepsilon}}=\mathbf{s . .} \dot{\boldsymbol{\sigma}}+\boldsymbol{\alpha} \dot{T}$

where $\mathbf{s}$ is the elastic compliance tensor of the single crystal.

And for the polycrystal:

$\dot{\mathbf{E}}=\mathbf{S} . \dot{\Sigma}+\mathbf{M} \dot{T}$

where $\mathbf{M}$ is the macroscopic thermal tensor and $\mathbf{S}$, the overall elastic compliance of the polycrystal. 

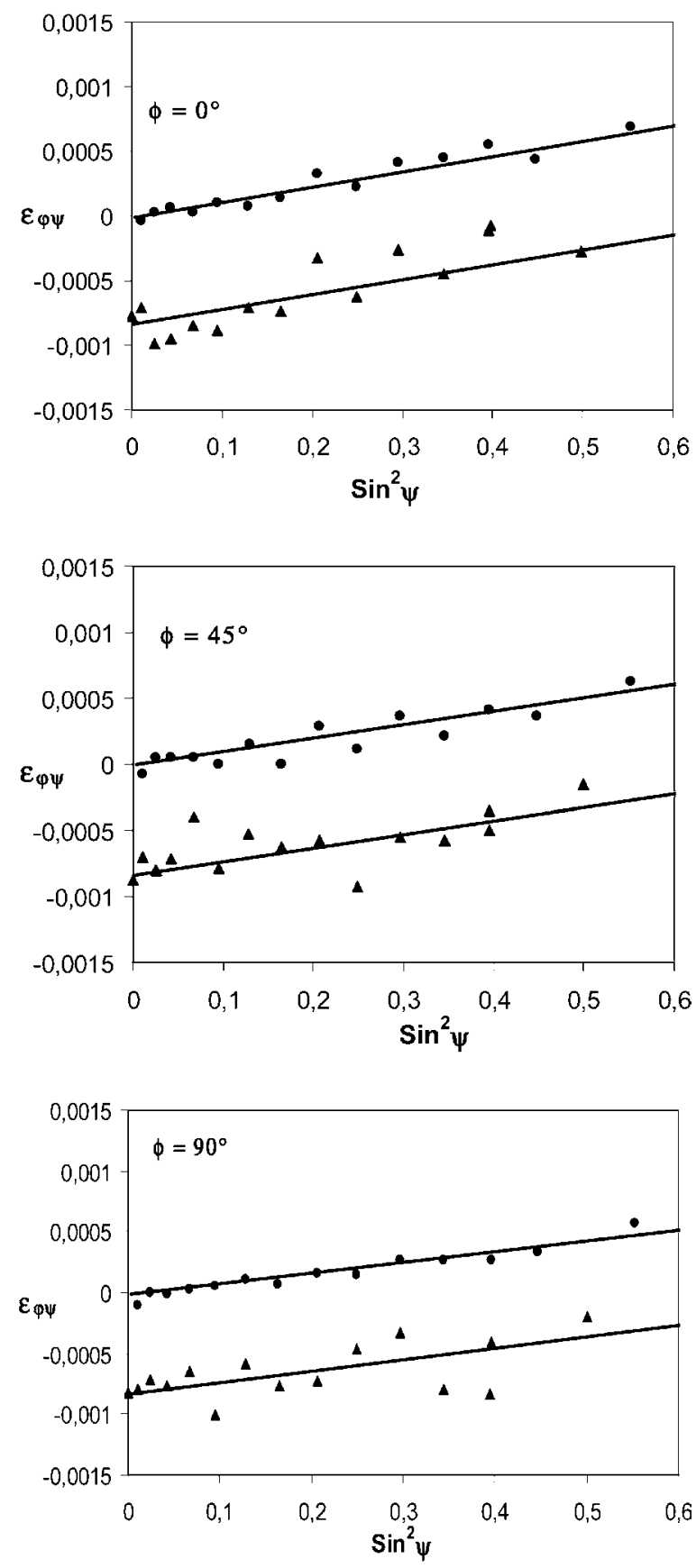

Fig. 5. Thermal residual strains vs $\sin ^{2} \Psi$ for (10.4) and (20.2) diffracting planes at room temperature. • (10.4) diffracting plane-experimental results; $\boldsymbol{\Delta}$ (20.2) diffracting planeexperimental results; — predicted results obtained using the self-consistent approach.
In terms of stress rate, we have the following equations:

$\dot{\boldsymbol{\sigma}}=\mathbf{c . .}(\dot{\boldsymbol{\varepsilon}}-\boldsymbol{\alpha} \dot{T})$ and $\dot{\boldsymbol{\Sigma}}=\mathbf{C} . .(\dot{\mathbf{E}}-\mathbf{M} \dot{T})$.

Strain and stress rate tensor are linearly linked by [10]:

$$
\begin{aligned}
& \dot{\boldsymbol{\sigma}}-\dot{\Sigma}=-\mathbf{C} . .\left(\mathbf{I}-\mathbf{S}^{e s h}\right) . . \mathbf{S}^{e s h-1} . .(\dot{\boldsymbol{\varepsilon}}-\dot{\mathbf{E}}) \\
& \quad=\text { B... }(\dot{\boldsymbol{\varepsilon}}-\dot{\mathbf{E}})
\end{aligned}
$$

where $\mathbf{S}^{\text {esh }}$ is the Eshelby tensor, calculated from the macroscopic compliances.

The determination of the thermo-elastic properties associated with the cooling process can be formulated as follows:

- The aggregate is subjected to thermal variations (cooling process) without external loading $(\dot{\Sigma}=0)$.

- No macro or mesoscopic plastic deformation takes place during the cooling process. Using Eqs. (3) and (4), the strain and stress in each grain induced by a uniform temperature increment are given by:

$\dot{\sigma}=\left(\mathbf{B}^{-1}-\mathbf{s}\right)^{-1} . .(\boldsymbol{\alpha}-\mathbf{M}) \dot{T}$

and

$\dot{\boldsymbol{\varepsilon}}=(\mathbf{c}-\mathbf{B})^{-1} . .(\mathbf{c} . . \alpha-\mathbf{B} . . \mathbf{M}) \dot{T}$.

The macroscopic thermal moduli $\mathbf{M}$ can also be calculated:

$\mathbf{M}=\left\langle(\mathbf{c}-\mathbf{B})^{-1} . . \mathbf{C}\right\rangle^{-1} . .\left\langle(\mathbf{c}-\mathbf{B})^{-1} . . \mathbf{c} . . \alpha\right\rangle$.

The unknowns are tensor $\mathbf{M}$ and tensor $\mathbf{C}$. $\mathbf{C}$ is determined using the well-known implicit equation

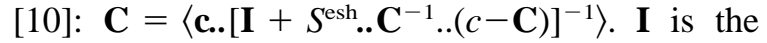
fourth order identity tensor. Furthermore, we can completely characterize the mechanical state of each grain for a temperature increment and describe the evolution of thermal residual stresses during a cooling process through Eqs. (5) and (6).

\subsection{Simulation of mechanical loading}

Plastic deformation takes place when a slip or a twinning system becomes active. Slip (or twinning) occurs if the resolved shear stress $\tau_{\mathrm{g}}$ on a system $\mathrm{g}$ is equal to the critical value $\tau_{\mathrm{c}}^{\mathrm{g}}$ and if the system remains loaded during the stress evolution: 
$\tau^{\mathrm{g}}=\mathbf{R}^{\mathrm{gr}} . \boldsymbol{\sigma}=\tau_{\mathrm{c}}^{\mathrm{g}}$ and $\mathbf{R}^{\mathrm{g}} . . \dot{\boldsymbol{\sigma}}=\tau_{\mathrm{c}}^{\mathrm{g}}$

where $\mathbf{R}^{\mathrm{g}}$ is the Schmidt tensor on a system $\mathrm{g}$.

The stress rate $\dot{\boldsymbol{\sigma}}$ is linearly linked to the total strain rate $\dot{\boldsymbol{\varepsilon}}$ by an elastoplastic consistent tangent moduli tensor $\mathbf{l}$ :

$\dot{\boldsymbol{\sigma}}=1 . . \dot{\varepsilon}$.

The tensor $\mathbf{I}$ has the form:

$\mathbf{l}=\mathbf{c} . . \mathbf{R}^{\mathrm{h}} . .\left(\mathbf{R}^{\mathrm{g}} . \mathbf{c} . . \mathbf{R}^{\mathrm{h}}+H^{\mathrm{gh}}\right)^{-1} . . \mathbf{R}^{\mathrm{g}} . \mathbf{c}$

where $\mathbf{c}$ is the elastic stiffness of the single crystal and $H$ the hardening matrix. This tensor depends on active systems, elastic properties, stress rate and deformation history of the grain.

At the macroscopic level, the relation between the macroscopic stress rate and strain rate is given by a similar relation as (8):

$\dot{\Sigma}=\mathbf{L} . . \dot{\mathbf{E}}$

$\mathbf{L}$ is the consistent tangent overall elastoplastic tensor.

The relations between local and global strains and stresses can be expressed through:

$\dot{\boldsymbol{\sigma}}=\mathbf{l} . .\left(\mathbf{I}+\mathbf{S}^{\text {esh }} . . \mathbf{L}^{-1} . . \Delta \mathbf{I}\right)^{-1} . . \mathbf{L}^{-1} . . \dot{\Sigma}$

and

$\dot{\boldsymbol{\varepsilon}}=\left(\mathbf{I}+\mathbf{S}^{\mathbf{e s h}} . . \mathbf{L}^{-1} . . \Delta \mathbf{I}\right)^{-1} . . \dot{\mathbf{E}}$

where $\Delta \mathbf{l}=\mathbf{l}-\mathbf{L}$.

$\mathbf{S}^{\text {esh }}$ is calculated assuming the grain as a spherical inclusion embedded to the homogeneous matrix. It is important to note that $\mathbf{S}^{\text {esh }}$ is calculated at each increment from the components of the tangent overall elastoplastic tensor $\mathbf{L}$.

The volume averages of the local stress and strain tensors must coincide with the overall strain and stress. After some algebraic calculations, these conditions give the overall elastoplastic tensor $\mathbf{L}$ as a weighted average of the mesoscopic tensor $\mathbf{l}$ :

$\mathbf{L}=\left\langle\mathbf{l} . .\left[\mathbf{I}+\mathbf{S}^{\text {esh }} . . \mathbf{L}^{-1} . . \Delta \mathbf{I}\right]^{-1}\right\rangle$.

Eq. (12) is a non-linear implicit equation because $\mathbf{S}^{\text {esh }}$ depends on the unknown $\mathbf{L}$. The Eshelby tensor is calculated by an integral equation [9] that taking into account the plastic anisotropy. This equation is solved by iteration. Once $\mathbf{L}$ is known, by specifying an overall stress or strain, the model can give the corresponding stress or strain tensors for each orientation. Therefore, we can describe the mechanical response of the polycrystal.

\section{Experimental and model results}

\subsection{Thermal stresses}

The first step of our work is to simulate the evolution of thermal residual stresses when the material is cooled from the annealing temperature to room temperature. We suppose the sample is stress-free at $923 \mathrm{~K} \mathrm{[1].} \mathrm{The} \mathrm{texture} \mathrm{was} \mathrm{introduced} \mathrm{in} \mathrm{the}$ model by a set of 1000 grains characterized by Euler angles and weights which represent their volume fraction. The single crystal elastic tensor used in the model are listed in Table 2 and the dilatation tensor at room temperature is [3]: $\alpha_{11}=\alpha_{22}=$ $5.70 \times 10^{-6} \mathrm{~K}^{-1}$ and $\alpha_{33}=11.40 \times 10^{-6} \mathrm{~K}^{-1}$.

We predict the evolution of thermal stresses as a function of temperature for different diffracting planes:

$-\{10 \overline{1} 4\},\{20 \overline{2} 2\}$ which are the planes studied by X-ray diffraction

$-\{0002\}$ and $\{10 \overline{1} 0\}$ are, respectively, the basal and the prismatic planes. These planes are interested to study because they generally have an opposite behaviour in term of strain values.

To analyse the influence of texture on the thermal response of the aggregate, two types of texture are considered in this work: an isotropic texture and the experimental one.

Although the thermal and elastic coefficients depend on temperature, several authors [2,5] use coefficients which are independent with temperature. To investigate this problem, we have considered successively two assumptions:

Table 2

Zirconium single-crystal stiffnesses [11]

\begin{tabular}{llllll}
\hline $\begin{array}{l}\text { C11 } \\
(\mathrm{GPa})\end{array}$ & $\begin{array}{l}\text { C12 } \\
(\mathrm{GPa})\end{array}$ & $\begin{array}{l}\text { C13 } \\
(\mathrm{GPa})\end{array}$ & $\begin{array}{l}\text { C33 } \\
(\mathrm{GPa})\end{array}$ & $\begin{array}{l}\text { C44 } \\
(\mathrm{GPa})\end{array}$ & $\begin{array}{l}\text { C66 } \\
(\mathrm{GPa})\end{array}$ \\
\hline 143.5 & 72.5 & 65.4 & 164.9 & 32.1 & 35.5 \\
\hline
\end{tabular}


- Independence of $c_{i j}$ and $\alpha_{i j}$ with temperature. The values are given in Table 2 .

- Variation of $c_{i j}$ and $\alpha_{i j}$ with temperature. The elastic constants measured by Fisher et al. [12] and the thermal expansion coefficients measured by Goldak et al. [13] are used. The temperature dependence of these constants can be described by a polynomial relation as proposed by Turner et al. [6].

Fig. 2 shows the residual stress evolution obtained using the elastic self consistent method with the assumptions discussed above. The temperature dependence of the elastic and thermal constants accounts for the non-linear evolution of the experimental curves observed by Mac Ewen et al. [3]. With the second assumption, this non-linear evolution can be obtained (the first shows a linear behaviour). Different strains values at room temperature have been obtained using the two assumptions. The difference is stronger for a textured material than for an isotropic one. It can reach as much as 18 to $28 \%$ or 40 to 400 microstrains according to the studied plane. Fig. 2 clearly shows the influence of crystallographic texture on strain evolution: the predicted values and even their sign vary strongly with the texture. The normal direction to $\{10 \overline{1} 4\}$ plane is in traction with an isotropic texture and in compression with the experimental texture. The prismatic plane is always under compressive strains while the basal plane is under tensile strains for any texture. These results agree with previous experimental results on rolled Zircaloy- 2 $[3,5]$. The diagonal components $\sigma_{22}$ and $\sigma_{33}$ of the stress tensor in every grain are plotted in Fig. 3 as a function of the angle $\Psi$ between the $c$-axis and the Normal Direction (ND). These values are expressed in crystal axes and correspond to values obtained at room temperature. The prismatic planes are in compression and the basal planes are in traction. Stress values are symmetric with respect to $\Psi=90^{\circ}$ because of orthotropic symmetry. These values are high: $30 \%$ of the grains have component $\sigma_{33}$ higher than $80 \mathrm{MPa}$ and component $\sigma_{22}$ lower than $-50 \mathrm{MPa}$. The grains with $\Psi$ close to $90^{\circ}$ have high stress values. This behaviour can be clearly seen on a stereographic projection of
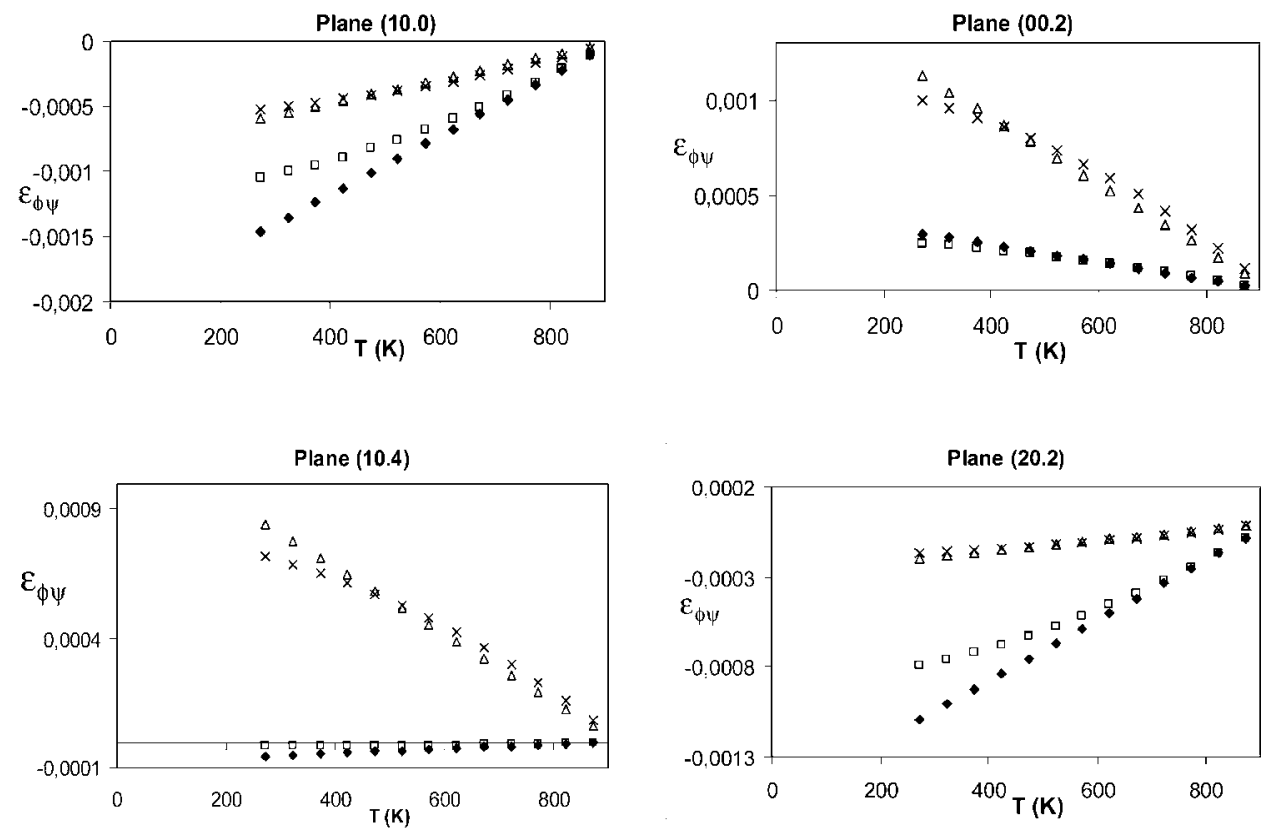

Fig. 2. Predicted thermal residual strains for different planes with $\psi=0^{\circ}$ and $\phi=0^{\circ}$ using: $\bullet$, experimental texture, independence of $c$ and $\alpha$ with $T ; \square$, experimental texture, dependence of $c$ and $\alpha$ with $T ; \triangle$, isotropic texture, independence of $c$ and $\alpha$ with $T$; $\times$, isotropic texture, dependence of $c$ and $\alpha$ with $T$. 


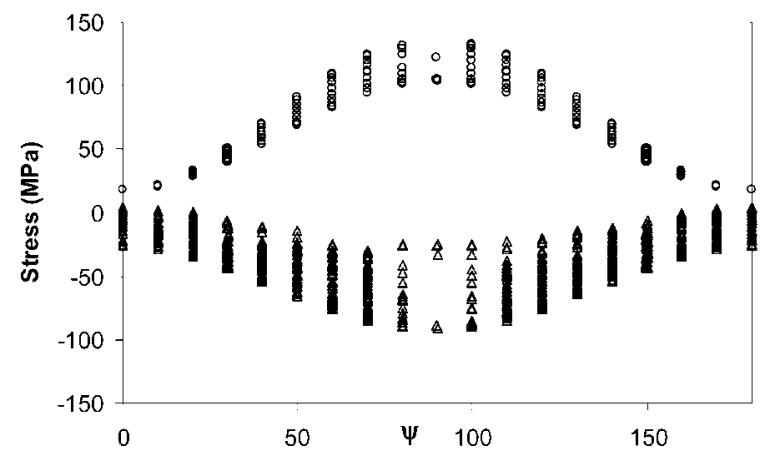

Fig. 3. Diagonal components $\sigma_{22}(\triangle)$ and $\sigma_{33}(\bigcirc)$ calculated in every grain as a function of the angle $\Psi$ at room temperature using experimental texture.

$\{0002\}$ pole (Fig. 4). The macroscopic thermal anisotropy explains this phenomenon. At macroscopic level, the contraction in the ND is greater than in the other directions. At mesoscopic level, grains are subjected to high contraction in the $c$ direction. Consequently, the grains with $\Psi$ close to $90^{\circ}$ induce more residual elastic strains to maintain compatibility between adjacent grains. Stress values obtained are always lower than the yield limit $(<200 \mathrm{MPa})$ and thus, it justifies the assumption made in Section 3 that thermal contractions do not introduce plastic deformation in the material

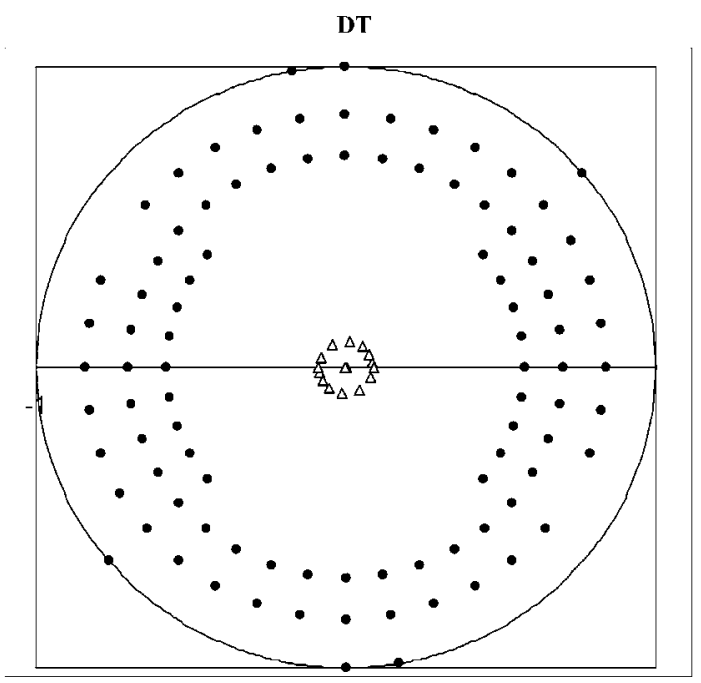

Fig. 4. Stereographic projection of (0002) pole for grains with $\sigma_{33}>100 \mathrm{MPa}(\bullet)$ or $\sigma_{22}<-30 \mathrm{MPa}(\triangle)$ (experimental texture). during the cooling process. The predicted thermal expansion coefficients of the polycrystal associated to the isotropic and experimental texture are respectively at room temperature: $\alpha_{11}=\alpha_{22}=\alpha_{33}=7.629 \times 10^{-6} \mathrm{~K}^{-1}$ and $\alpha_{11}=$ $5.163 \times 10^{-6} \mathrm{~K}^{-1}, \alpha_{22}=5.463 \times 10^{-6} \mathrm{~K}^{-1}, \alpha_{33}=$ $7.031 \times 10^{-6} \mathrm{~K}^{-1}$.

Using the self consistent formulation, we simulated residual thermal strains versus $\sin ^{2} \Psi$ for the $\{10 \overline{1} 4\}$ and $\{20 \overline{2} 2\}$ diffracting planes. These strains can be represented by a straight line $\mathbf{A}+$ $\mathbf{B} \sin ^{2} \Psi$ (Fig. 5). The predicted results are presented in Table 3.

The values of $A$ must be independent of $\phi$. This fact enables the estimation of the numerical precision of our calculations. We can express $A$ values in term of stress with the XEC (determinated by an elastic self consistent model for an isotropic material). The standard deviation varies from 1.6 to $2.1 \mathrm{MPa}$ with the studied plane. The choice of 1000 orientations is justified by this low dispersion.

Predicted results were compared with experimental data using X-ray diffraction method. The experimental strain values are determined using the following expression:

$\varepsilon_{\varphi \psi}=-\frac{1}{2} \operatorname{cotang} \theta_{0}\left(2 \theta_{\varphi \psi}-2 \theta_{0}\right)$

where $\theta_{0}$ is the Bragg angle for a stress-free specimen and $\theta_{\phi \Psi}$ is the measured Bragg angle. Consequently, it was necessary to determinate the stressfree lattice parameters of the sample to calculate the thermal strains. Because of strains induced by thermal anisotropy, these parameters cannot be measured directly. One solution [2] is to find the stress-free parameters $a_{0}$ and $c_{0}$ which minimize the difference between experimental data and predict strains for the two studied planes. With this method, we found the following values:

$$
\begin{aligned}
& a=3.2365 \AA \text { and } c=5.1498 \AA \\
& \text { or } \\
& 2 \theta_{0}\{10 \overline{1} 4\}=156.240^{\circ} \text { and } 2 \theta_{0}\{20 \overline{2} 2\}=136.896^{\circ} .
\end{aligned}
$$

For the sake of comparison, the values obtained with the best fit by Ortiz et al. for Zirconium- $\alpha$ are: $a=3.2325 \AA$ and $c=5.1464 \AA$.

The differences between these values are of the 
Table 3

Predicted $A$ and $B$ coefficients for $\{10 \overline{1} 4\}$ and $\{20 \overline{2} 2\}$ diffracting planes

\begin{tabular}{lcccccc}
\hline \multicolumn{1}{c}{ Plane $\{20 \overline{2} 2\}$} & \multicolumn{3}{c}{ Plane $\{10 \overline{1} 4\}$} \\
\hline & $\phi=0^{\circ}$ & $\phi=45^{\circ}$ & $\phi=90^{\circ}$ & $\phi=0^{\circ}$ & $\phi=45^{\circ}$ & $\phi=90^{\circ}$ \\
\hline$A\left(\times 10^{3}\right)$ & -0.84200 & -0.84225 & -0.83294 & -0.02101 & -0.02388 & -0.03577 \\
$B\left(\times 10^{3}\right)$ & 1.15022 & 1.03106 & 0.93470 & 1.17819 & 1.00939 & 0.87753 \\
$A(\mathrm{MPa})$ & 231.9 & 231.4 & 228.8 & 5.8 & 6.5 & 9.8 \\
\hline
\end{tabular}

order of $4 \times 10^{-3} \AA$. It should be noted that, except for the stress-free lattice parameters $a$ and $c$, not any parameter of the model was fit on experiments. Fig. 5 shows the experimental (solid circles and triangles) and predicted (full line) thermal strains versus $\sin ^{2} \Psi$ curves for $\{10 \overline{1} 4\}$ and $\{20 \overline{2} 2\}$ diffracting planes. Table 4 gives the experimental coefficients $A$ and $B$ expressed in terms of strain and stress. The quadratic difference between the predicted and experimental values is $24 \mathrm{MPa}$ and $11 \mathrm{MPa}$ for $\{20 \overline{2} 2\}$ and $\{10 \overline{1} 4\}$ planes. Compared with the uncertainty on experiments (16 MPa) and modelization (2 $\mathrm{MPa})$, this difference is reasonable.

If we consider an isotropic texture, stress values in each grain are: $35 \%$ of the grains have component $\sigma_{33}$ higher than $100 \mathrm{MPa}$ and component $\sigma_{22}$ lower than $-50 \mathrm{MPa}$. Stress values vary weakly with $\Psi$ because of the isotropic texture. The slope of $\varepsilon_{\phi \Psi}$ versus $\sin ^{2} \Psi$ is close to 0 . The effect of thermal stresses is only represented by a translation of the interceipt of the straight lines: $\mathbf{B}=$ 0.00079 for $\{10 \overline{1} 4\}$ plane and -0.00028 for $\{20 \overline{2} 2\}$ plane.

It should be noted that the slope of $2 \theta$ (or $\varepsilon_{\phi \Psi}$ ) versus $\sin ^{2} \Psi$ is not related to any macroscopic stress, it is a projection of mesoscopic stresses.
Only to give an idea of the magnitude, it can be said that it corresponds to $90 \mathrm{MPa}(\approx 35 \%$ of the yield stress) at room temperature. In conclusion, the thermal stresses at room temperature cannot be neglected and can play an important role on the elastoplastic transition (see next section).

\subsection{Mechanical stresses}

The second part of this work is the simulation of the mechanical response of rolled $\mathrm{Zr}-\alpha$ when it is subjected to uniaxial loading along the rolling and the transverse directions.

Generally, deformation in Zirconium alloys can occur by either slip or twinning. In such materials, microstructural observations show that prismatic glide $\{10 \overline{1} 1\}\langle 11 \overline{2} 0\rangle$ is the most active deformation mode $[14,15]$. First-order pyramidal slip $\{10 \overline{1} 1\}\langle 11 \overline{2} 3\rangle$ and basal slip $\{0002\}\langle 11 \overline{2} 0\rangle$ are considered as secondary slip mode $[15,16]$. At room temperature, twinning in Zirconium is most frequently observed to occur on the $\{10 \overline{1} 2\},\{11 \overline{2} 1\}$ and $\{11 \overline{2} 2\}$ planes $[17,18]$.

In the model, critical resolved shear stresses (CRSS) and the hardening law for slip and twinning are considered as controlling parameters.

Table 4

Experimental $A$ and $B$ coefficients for $\{10 \overline{1} 4\}$ and $\{20 \overline{2} 2\}$ diffracting planes

\begin{tabular}{lccccrr}
\hline \multicolumn{1}{c}{ Plane $\{20 \overline{2} 2\}$} & \multicolumn{3}{c}{ Plane $\{10 \overline{1} 4\}$} \\
\hline & $\phi=0^{\circ}$ & $\phi=45^{\circ}$ & $\phi=90^{\circ}$ & $\phi=0^{\circ}$ & $\phi=45^{\circ}$ & $\phi=90^{\circ}$ \\
\hline$A\left(\times 10^{3}\right)$ & -0.90343 & -0.78922 & -0.81571 & -0.02992 & -0.04344 & -0.06561 \\
$B\left(\times 10^{3}\right)$ & 1.65645 & 0.85342 & 0.80209 & 1.28388 & 1.03634 & 1.00242 \\
$A(\mathrm{MPa})$ & 248.2 & 216.8 & 224.1 & 8.2 & 11.9 & 18.1 \\
\hline
\end{tabular}


Their values depend on many factors (oxygen content, grain size, temperature, etc.) and they are unknown. Consequently, they are determined through a fitting of the predicted behaviour on the experimental one.

The initial CRSS are taken as identical for all systems of a system type. The deformation systems introduced in the model are assumed to be: prismatic slip, first-order pyramidal slip and $\{10 \overline{1} 2\}$ twinning. In this work, we considered a linear hardening law [7], the coefficient $H^{\text {gr }}$ is equal to $H^{\mathrm{gg}}$ for any deformation modes $r: \dot{\tau}^{\mathrm{g}}=H^{\mathrm{g}} \sum_{r} \dot{\gamma}^{r}$, i.e. latent hardening is equal to the self-hardening. The CRSS obtained are: $\tau=118 \mathrm{MPa}$ for prismatic mode, $\tau=190 \mathrm{MPa}$ for first-order pyramidal mode and $\tau=240 \mathrm{MPa}$ for tensile twinning. The hardening coefficients were found to be 69 MPa for prismatic slip, $150 \mathrm{MPa}$ for pyramidal slip and $240 \mathrm{MPa}$ for twin mode. The CRSS for the prismatic systems is determined to reproduce the experimental yield stress in the RD. The CRSS values are adjusted to give a good description of the tensile test performed in the RD of the sheet. Experimental observations $[17,18]$ show that twinning does not make a large contribution to plastic deformation. A CRSS value of $240 \mathrm{MPa}$ ensure that their activity is lower than prism or pyramidal activity. The hardening coefficients are chosen to be consistent with the final experimental slope of the curve in the RD of the sample. The same parameters are taken for the simulation of the tensile test in the TD. We have considered two assumptions in the simulations: loading with thermal stresses and without thermal stresses. Fig. 1 shows the predicted stress-strain curves for a tensile test in rolling and transverse directions.

The experimental tests show several differences between the two directions: the yield stress is higher, the elastoplastic transition is faster and the curve shows a plateau at $0.6 \%$ strain in the transverse direction. The measured yield stresses are $205 \mathrm{MPa}$ and $290 \mathrm{MPa}$ (stress differential of $85 \mathrm{MPa}$ ) in the $\mathrm{RD}$ and TD, respectively. The corresponding Young's modulus are $93 \mathrm{GPa}$ and $96 \mathrm{GPa}$. These values are similar because of the low elastic anisotropy. With the model developed here, the pre- dicted Young's modulus are $93 \mathrm{GPa}$ and $95 \mathrm{GPa}$ in agreement with the experimental ones. The stress differential can be attributed to thermal stresses. This fact has been already analysed by Mac Ewen et al. [3] on Zircaloy-2. The predicted yield stresses are $205 \mathrm{MPa}$ and $275 \mathrm{MPa}$ in the RD and TD, respectively. When no thermal stresses are assumed, the model predicts a low stress differential $(<15 \mathrm{MPa})$ which could be attributed to texture.

For the test along the RD, the elastoplastic transition starts with the activation of prismatic slip. At $0.7 \%$ strain, pyramidal slip is activated and when the deformation reaches $1.3 \%$ deformation, the polycrystal is fully plastic. This sequence explains correctly the experimental loading slope. At $1 \%$ deformation, the proportions of activated systems are: $83 \%$ for prismatic mode, $16.5 \%$ for pyramidal slips and $0.5 \%$ for tensile twinning. Finally, we obtained a reasonable distribution in agreement with the experimental observations: prismatic glide is the main active mechanism, pyramidal slip is a secondary slip mode and twinning contributes very little to plastic deformation.

When tension is simulated in the TD, the yield stress is higher and the plastic deformation begins by the activation of prismatic slip. At $0.4 \%$ strain, pyramidal systems become active and the material is fully plastic at about $0.9 \%$. At $1 \%$, the distribution of activated systems is: $75 \%, 24.2 \%$ and $0.8 \%$ for the prismatic, pyramidal and tensile twin respectively. The model fails to reproduce correctly the plateau in the curve. Turner et al. [6] attributed this part to the relaxation of stresses by twinning, however they did not introduce it in their model. In our model, this phenomenon is incorporated, but we found that plastic deformation due to twinning is less than $1.0 \%$ and this result is coherent with experimental observations. Consequently, it seems clearly that twinning cannot explain the plateau in the curve in the TD. If we take lower values of CRSS or hardening coefficients that does not modify this tendency.

In Fig. 6, the stress axis orientation is plotted in the stereographic basal projection of the hexagonal lattice for each grain. The grains in which prismatic slip was first activated, have the angle between stress axis and pole of basal plane close to $90^{\circ}$ (the Schmidt factor becoming maximum). For pyramidal 


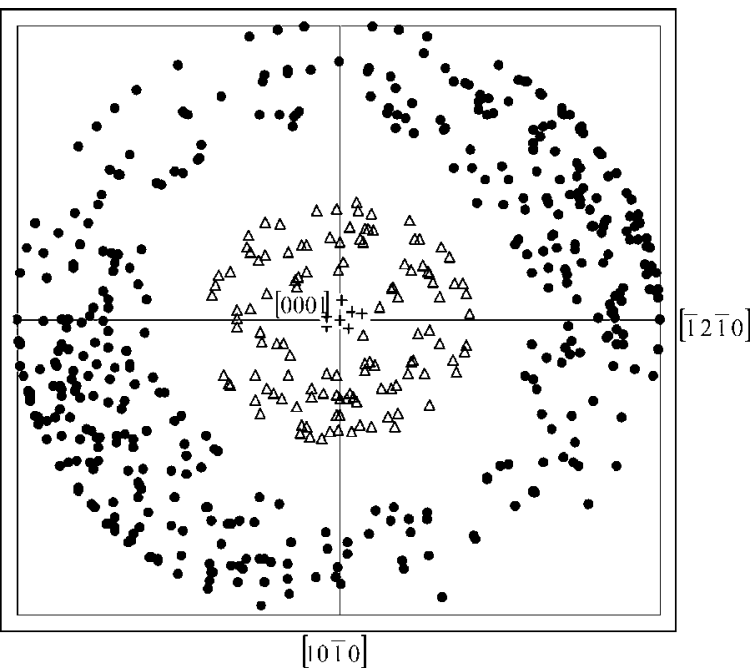

Fig. 6. Stress axis orientation in a basal stereographic projection of the hexagonal lattice with traction in RD. $\bullet$ Grains in which prismatic mode was first activated; $\triangle$ grains in which pyramidal mode was first activated; + grains in which twinning mode was first activated.

mode, this angle is close to $45^{\circ}$. Twinning may occur when this axis is quasi parallel to the $c$-axis. This fact explains why the CRSS and hardening parameters of twinning have a very low influence on the macroscopic behaviour: twinning concerns only a small fraction of specifically oriented grains. The grains which have the highest value of strain with the lowest value of stress correspond to grains with $c$-axis normal to the stress axis.

\section{Conclusions}

In this study, the thermal residual stresses in a rolled $\mathrm{Zr}-\alpha$ using a self-consistent model have been evaluated and compared with X-ray diffraction results. A good agreement between the two methods has been obtained. The residual stresses due to thermal anisotropy are rather important (equivalent to $35 \%$ of the yield stress) and consequently they play an important role on the elastoplastic transition. The stress-free lattice parameters of our sample were determined with the model and we found $a=$ $3.2365 \AA$ and $c=5.1498 \AA$.

An elastoplastic self-consistent approach has been developed for modelling the mechanical response of rolled $\mathrm{Zr}$ under uniaxial loading. We showed that the thermal stresses can explain the stress differential between RD and TD of the sample and that the elastoplastic transition can be explained by the texture and the internal stresses. This study has enable us to check the capacity of our model to simulate the internal stresses in rolled $\mathrm{Zr}-\alpha$ and explains its behaviour. Next step will be to verify the assumptions on the deformation mode activated in our calculation. For that, samples will be subjected to uniaxial loading with different total strain along the rolling and the transverse directions. Stress measurements with diffraction techniques on these samples and simulation will allow a better prevision and interpretation of the mechanical behaviour of the material. A similar study on an hexagonal material (Ti- $\alpha$ ) with $c / a$ close to Zirconium value (1.5873) is also beginning.

\section{References}

[1] Ortiz M, Pochettino AA. In: Denis S, et al., editors. Fourth European Conference on Residual Stresses, vol. 2 1996:971-6.

[2] Ortiz M, Pochettino AA. J Nucl Mat 1996;229:65-72.

[3] Mac Ewen SR, Tomé C, Faber J. Acta Mater 1989;37:979-89.

[4] Mac Ewen SR, Christodoulou N, Salinas-Rodriguez A. Metall Trans 1990;21A:1083-95.

[5] Turner PA, Tomé CN. Acta Mater 1994;42:4143-59.

[6] Turner PA, Christodoulou N, Tomé CN. Int J Plasticity 1995;11(3):251-65.

[7] Pang JWL, Holden TM, Turner PA, Masson TE. Acta Mater 1999;47:373-83.

[8] Hill R. J Mech Phys Solids 1965;13:89-101.

[9] Lipinski P, Berveiller M. Int J Plasticity 1989;5:149-72.

[10] Kocks UF, Tomé CN, Wenk HR. Texture and anisotropy. Cambridge: Cambridge University Press, 1998.

[11] Simmons G, Wang H. Single crystal elastic constants and calculated aggregate properties. Cambridge, (MA): MIT Press, 1971.

[12] Fisher ES, Renken C. Phys Rev 1964;135:484-94.

[13] Goldak J, Lloyd LT, Barrer CS. Phys Rev 1966;144(2):478-83.

[14] Pochettino AA, Gannio N, Vial Edwards C, Penelle R. Scripta Metall 1992;27:1859-63.

[15] Tenckhoff E. Deformation mechanisms, texture, and anisotropy in zirconium and zircaloy. STP 966, 1916 Race Street, Philadelphia, PA 19103, 1988.

[16] Akhtar A. Acta Metall 1973;21:1-11.

[17] Akhtar A. Met Trans 1975;6A:1105-13.

[18] Akhtar A. J Nucl Mat 1973;47:79-86. 